\title{
Mudanças no Código Florestal e seu impacto na ecologia e diversidade dos mamíferos no Brasil
}

\author{
Mauro Galetti ${ }^{1,6}$, Renata Pardini ${ }^{2}$, José Maurício Barbanti Duarte ${ }^{3}$, \\ Vera Maria Ferreira da Silva ${ }^{4}$, Alexandre Rossi ${ }^{1}$ \& Carlos Augusto Peres $^{5}$ \\ ${ }^{1}$ Departamento de Ecologia, Universidade Estadual Paulista - UNESP, \\ CP 199, CEP 13506-900, Rio Claro, SP, Brasil \\ ${ }^{2}$ Departamento de Zoologia, Universidade de São Paulo - USP, \\ Rua do Matão, Travessa 14, n. 101, CEP 05508-090, São Paulo, SP, Brasil \\ ${ }^{3}$ Departamento de Zootecnia, \\ Universidade Estadual Paulista - UNESP, CEP 14884-900, Jaboticabal, SP, Brasil \\ ${ }^{4}$ Laboratório de Mamíferos Aquáticos, Instituto Nacional de Pesquisas da Amazônia - INPA, \\ CP 471, CEP 69081-001, Manaus, AM, Brasil \\ ${ }^{5}$ Centre for Ecology Evolution and Conservation, School of Environmental Sciences, \\ University of East Anglia, Norwich, NR4 7TJ, UK \\ ${ }^{6}$ Autor para correspondência: Mauro Galetti,e-mail: mgaletti@rc.unesp.br
}

GAletti, M., PARDini, R., DUARTE, J.M.B., SilVA, V.M.F., ROSSI, A. \& PERES, C.A. Forest legislative changes and their impacts on mammal ecology and diversity in Brazil. Biota Neotrop. 10(4): http://www.biotaneotropica.org.br/v10n4/en/abstract?article+bn00710042010.

\begin{abstract}
Forest ecosystems within Brazil host one of the highest levels of mammalian diversity on Earth, much of which within legally required forest set-asides in private landholdings. The Legal Reserves (RLs) and Permanent Protected Areas (APPs) of the Brazilian Forest Code provide an important strategy to maintain this diversity. Yet a proposed amendment to Brazil's 1965 forestry code would reduce protection of Brazil's forests, including the Amazon and the Atlantic forest, and bring irreversible detrimental effects to mammal diversity. Mammals are key components of forest ecosystem, providing important environmental services as pollinators, seed dispersers and ecosystem engineers. The local extinction of some species will negatively affect forest ecosystem service provisioning throughout the country. Another important effect of forest conversion within private properties, should the proposed changes happen, will be the emergence of new diseases, bringing serious public health problems in Brazil.

Keywords: edge effects, flooded forests, riverine forests, corridors, landscape connectivity, human-dominated landscapes, ecosystem services.
\end{abstract}

GALETTI, M., PARDINI, R., DUARTE, J.M.B., SILVA, V.M.F., ROSSI, A. \& PERES, C.A. Mudanças no Código Florestal e seu impacto na ecologia e diversidade dos mamíferos no Brasil. Biota Neotrop. 10(4): http://www.biotaneotropica.org.br/v10n4/pt/abstract?article+bn00710042010.

Resumo: Os ecossistemas florestais do Brasil abrigam um dos mais altos níveis de diversidade de mamíferos da Terra, e boa parte dessa diversidade se encontra nas áreas legalmente protegidas em áreas de domínio privado. As reservas legais (RLs) e áreas de proteção permanente (APPs) representam estratégias importantes para a proteção e manutenção dessa diversidade. Mudanças propostas no Código Florestal certamente trarão efeitos irreversíveis para a diversidade de mamíferos no Brasil. Os mamíferos apresentam papéis-chave nos ecossistemas, atuando como polinizadores e dispersores de sementes. A extinção local de algumas espécies pode reduzir os serviços ecológicos nas RLs e APPs. Outra consequência grave da redução de áreas de vegetação nativa caso a mudança no Código Florestal seja aprovada será o aumento no risco de transmição de doenças, trazendo sério problemas a saúde pública no Brasil.

Palavras-chave: efeito de borda, várzea, matas ciliares, corredores, conectividade de paisagens, paisagens antropizadas, serviços ecossistêmicos. 


\section{Introdução}

Cerca de um terço dos mamíferos se encontram ameaçados de extinção em nosso planeta, sendo que a sobre-exploração resultante da caça, perseguição e comércio ilegal, introdução de espécies exóticas, e principalmente a perda e degradação de hábitat, são as principais ameaças às espécies (Schipper et al. 2008). O Brasil detém a segunda maior diversidade de mamíferos do mundo (Vié et al. 2009) com 652 espécies nativas (Reis et al. 2006), das quais a grande maioria depende de habitats florestais. O futuro dessa diversidade dependerá de como o homem do século XXI pretende utilizar os recursos do planeta.

A grande maioria das espécies de mamíferos neotropicais depende das áreas de vegetação nativa preservadas para se manter em paisagens dominadas pelo homem, com exceção de algumas poucas espécies que proliferam e são capazes de manter populações em ambientes urbanos ou agrícolas, como alguns roedores e marsupiais (Umetsu \& Pardini 2007) ou morcegos (Sazima et al. 1994). Os mamíferos desempenham papéis fundamentais na dinâmica dos ecossistemas, seja como predadores de topo de cadeia alimentar que regulam herbívoros generalistas (Terborgh et al. 2001), seja como polinizadores (Sazima et al. 1982), dispersores e predadores de sementes que influenciam a regeneração da vegetação (Jordano et al. 2006) ou provendo recursos que mantêm espécies que exercem funções ecológicas importantes (Nichols et al. 2009). Por outro lado, algumas espécies de mamíferos generalistas que tendem a proliferar nos ambientes antrópicos, podem se tornar importantes reservatórios de doenças infecciosas ao homem (Owen et al. 2010) ou pragas na agricultura (Stenseth et al. 2003). Portanto, se quisermos manter a sustentabilidade dos ambientes naturais nas paisagens modificadas pelo homem, certamente temos que levar em conta o papel dos mamíferos nos ecossistemas.

A preservação de remanescentes de habitats nativos nos diferentes biomas brasileiros depende da manutenção de ecossistemas naturais, seja em áreas de domínio público, como em muitas Unidades de Conservação (UCs) e territórios indígenas, seja em áreas de domínio privado, como algumas das que devem ser protegidas inclusive por proprietários particulares como define a Lei 4.771 de 15 de setembro de 1965, mais conhecida como Código Florestal (CF).

Se por um lado cientistas apontam a importância do CF em proteger a biodiversidade em áreas de domínio privado como Áreas de Proteção Permanente (APPs) e Reservas Legais (RLs) (Metzger 2010); de outro lado, setores da agricultura e pecuária tem sistematicamente tentado modificar ou protelar a aplicação dessa lei, visando diminuir ou desfigurar, e em alguns casos eliminar, as APPs ou RLs. As recentes propostas de modificação do CF, especialmente aquelas que venham a reduzir a área de APP e RL trarão sérios impactos negativos nos serviços ecossistêmicos que essas áreas prestam; como os recursos renováveis e não renováveis bióticos (proteção da biodiversidade), geológicos, hídricos (proteção de nascentes e corpos d'água), atmosféricos (sequestro de carbono) e recursos da terra (Chan et al. 2006). Neste artigo, nós procuramos analisar estas questões, discutindo possíveis efeitos que permitem sustentar ou refutar as reformulações propostas no Código Florestal. Dado a nossa especialidade, nós vamos nos ater as discussões de alguns pontos em relação às mudanças nas APPs e RLs para os quais a ecologia de mamíferos tem importantes contribuições.

Em relação às mudanças nas APPs discutiremos os impactos das seguintes propostas de mudança no CF: 1) redução da largura das APPs, 2) acesso de pessoas e animais domésticos as APPs e o uso sustentável dessas áreas, 3) eliminação de APPs abrangendo topos de morros, montanhas e serras e 4) eliminação de áreas de várzeas como APP. No que tange as RLs: 1) eliminação da exigência de RLs para pequenas propriedades rurais, e 2) descaracterização da composição florística das RLs com o plantio de espécies exóticas.

\section{Impacto nas Áreas de Preservação Permanente (APP)}

\section{Redução da largura das APP}

O atual Código Florestal estipula uma série de larguras mínimas de áreas de proteção ao longo de cursos d'água, reservatórios e nascentes. No entanto, a menor largura em vigor hoje é de $30 \mathrm{~m}$ de cada lado dos rios e córregos d'água. Para a maioria das espécies de plantas e vertebrados, a faixa de no mínimo $30 \mathrm{~m}$ ao lado de cursos d'água geralmente não é suficiente para assegurar a manutenção dessa biodiversidade em longo prazo e promover a conectividade da paisagem por dois motivos principais: 1) efeito de borda e 2) redução de hábitat (Metzger 2010).

Efeito de Borda. O efeito de borda é resultante das rápidas alterações estruturais e micro-climáticas associadas à transição abrupta entre dois ecossistemas adjacentes (Murcia 1995). Essas mudanças podem ser percebidas por organismos florestais até $400 \mathrm{~m}$ em relação à borda mais próxima. Porém a maioria dos efeitos negativos, como invasão de espécies generalistas e invasoras, aumento da temperatura e diminuição da umidade relativa do ar, ressecamento do solo, alta mortalidade de árvores, redução da altura do dossel, entre outros efeitos deletérios à biota, ocorrem principalmente nos primeiros $100 \mathrm{~m}$ de distância da borda (Laurance et al. 2002). Além disso, estudos que avaliam a erosão do solo em margens de rios sugerem que larguras mínimas de $52 \mathrm{~m}$ ao lado de corpos d'água sejam mantidas para retenção de $>50 \%$ do total de sedimentos nas margens (Sparovek et al. 2002).

A redução das larguras das APPs proposta na reformulação do código florestal acarretará um efeito de borda mais acentuado, levando a uma maior taxa de predação de sementes (Fleury \& Galetti 2006), maior recrutamento de espécies ruderais (Tabarelli \& Peres 2002), aumento da mortalidade de árvores de grande porte (Laurance et al. 1997) que são especialmente importantes na produção de frutos para os mamíferos, aves e peixes (Reys et al. 2009) e, consequentemente, menor diversidade de espécies de aves e mamíferos florestais (Lees \& Peres 2008). O empobrecimento das APPs afetará também a produtividade primária da floresta (flores, frutos e folhas), tendo efeitos negativos sobre várias espécies de mamíferos associados às matas ciliares, como bugios (Alouatta spp.), macacos-prego (Cebus spp.), mãos-pelada (Procyon cancrivorus), quatis (Nasua nasua), queixadas (Tayassu pecari), pacas (Cuniculus paca), assim como espécies semi-aquáticas, como lontras (Lontra longicaudis), ariranhas (Pteronura brasiliensis), ratões-do-banhado (Myocastor coypus), capivaras (Hydrochoerus hydrochaeris) e cuícas d'água (Chironectes minimus) e aquáticas como o peixe-boi (Trichechus inunguis).

Redução de hábitat. A redução das APPs terá implicação direta na redução de habitats disponível para os mamíferos. Uma das teorias mais conhecidas na biologia da conservação é a relação espécie-área (MacArthur \& Wilson 1967), que enfatiza a relação não-linear entre a área do remanescente de vegetação nativa e o número de espécies que esta área pode sustentar.

As APPs são fundamentais para a persistência de populações de mamíferos, pois criam na paisagem corredores que permitem a conectividade de populações previamente isoladas. Esse estreitamento generalizado das APPs, porém, poderá levar a ruptura da conectividade garantida pelos corredores de florestas ripárias. Várias espécies de mamíferos são relutantes em atravessar áreas abertas como plantações ou pastagens, dado o risco elevado de mortalidade, causado pela 
predação por cães domésticos (Galetti \& Sazima 2006), perseguição pela população humana (Baker et al. 2008) e atropelamentos em estradas (Coelho et al. 2008). Outras não são tolerantes aos distúrbios antrópicos e dependem diretamente das matas ciliares, como é o caso das ariranhas (Rosas et al. 2008) e outras espécies aquáticas ou semi-aquáticas.

Além de favorecer o deslocamento de indivíduos entre manchas de vegetação nativa, os corredores servem como habitat, abrigando populações de espécies nativas de mamíferos de menor porte (Lima \& Gascon 1999), mas não de espécies exóticas ou invasoras (Naxara 2008). Pela influência direta dos rios e córregos e consequente minimização dos efeitos microclimáticos associados a bordas, os corredores ripários associados às RLs parecem ser particularmente adequados como habitat para espécies de pequenos mamíferos florestais (Naxara 2008). Desta maneira, a presença de corredores em paisagens fragmentadas leva ao aumento da abundância e riqueza destas espécies em pequenos fragmentos (Pardini et al. 2005), contribuindo para a persistência destas populações no médio e longo prazo. No cerrado, as matas de galerias são fundamentais como abrigos para os pequenos mamíferos, pois funcionam como refúgios para as espécies durante as queimadas (Vieira \& Marinho-Filho 1998). O mesmo ocorre em áreas incendiadas sazonalmente, como pastos e plantações de cana de açúcar, onde a mata ciliar é usada como abrigo e refúgio pelos mamíferos.

Portanto, a redução da largura das APPs deve acelerar o empobrecimento biológico dessas áreas e acarretar num maior número de extinções locais de espécies. O estreitamento das APPs ripárias implica também na perda ou degradação de serviços ecossistêmicos florestais, tais como a proteção e regulagem dos corpos d'água e a manutenção da conectividade da paisagem mesmo para as espécies florestais que não estão diretamente associadas a ambientes ripários.

\section{Acesso de pessoas e animais domésticos as APPs}

A permissão de acesso das APPs a animais domésticos afetará o recrutamento das espécies florestais e modificará substancialmente o sub-bosque dessa vegetação (Ramirez-Marcial et al. 2001). Estudos mostram que ocorre um aumento na predação de sementes em áreas pastadas por gado, com aumento da abundância de formigas que se alimentam de sementes (Meeson et al. 2002). Muitas espécies de marsupiais e roedores que ocupam o sub-bosque poderão ser extintos localmente caso o pisoteio por animais domésticos suprima essa vegetação (Vieira \& Monteiro-Filho 2003). Na Amazônia meridional, por exemplo, o pisoteio crônico e excesso de herbivoria pelo gado bovino e bubalino em pastos adjacentes estão associados a uma degradação da estrutura dos remanescentes de florestas ripárias, assim como um empobrecimento da diversidade de aves e mamíferos (Lees \& Peres 2008).

Como os animais domésticos (Pereira et al. 2000), animais selvagens (Szabó et al. 2007, 2009) e o próprio homem compartilham inúmeras enfermidades, o acesso livre desses três elementos às APPs facilitará a transmissão de doenças, podendo causar grandes danos à fauna (Szabó et al. 2003, Tiemann et al. 2005), problemas econômicos para as criações animais (Araújo Júnior et al. 2010) e o surgimento de zoonoses emergentes em humanos (Machado et al. 2006).

\section{Eliminação de topos de morros, montanhas e serras como APPs}

As zonas montanhosas são habitat de uma grande diversidade de mamíferos, muitos ameaçados de extinção e endêmicos. Por exemplo, o sagui da serra, Callithrix aurita, possui suas maiores populações em zonas de encosta da Serra do Mar (D. Norris et al. dados não publicados). O veado-mão-curta (Mazama nana), espécie ameaçada típica da Floresta Ombrófila Mista do Sul do Brasil, ocupa ambientes montanhosos desse bioma (Abril et al. 2010). Hoje a espécie sobrevive em poucos remanescentes desse habitat que sobraram nas encostas e serras do Paraná e Santa Catarina, e seria seriamente impactado pela modificação desse ambiente. O mesmo acontece com o veado-mateiro-pequeno (Mazama bororo) da Floresta Ombrófila Densa das Serras do Mar e de Paranapiacaba (Vogliotti \& Duarte 2009, 2010).

Essas áreas não apenas abrigam uma alta diversidade de espécies de mamíferos endêmicos, como também servem de trampolins ecológicos e corredores. Algumas espécies de mamíferos, como os queixadas (Tayassu pecari), apresentam grandes deslocamentos sazonais ao longo de gradientes altitudinais em resposta aos padrões de frutificação de algumas espécies de frutos carnosos (M. Galetti dados não publicados).

\section{Eliminação da várzea como $A P P$}

Hess et al. (2003) estimou que $17 \%$ de uma área de 1.77 milhões de $\mathrm{km}^{2}$ da Amazônia central é sazonalmente inundada e que as várzeas constituem quase $70 \%$ dessa área inundável. As comunidades de mamíferos das florestas sazonalmente inundadas na Amazônia são bastante diferenciadas das comunidades de mamíferos de florestas de terra firme adjacentes, em termos de composição, abundância e biomassa, com um predomínio de mamíferos arborícolas (Haugaasen \& Peres 2005). Além disso, várias espécies de primatas, roedores e marsupiais ocorrem preferencialmente ou exclusivamente nas florestas de várzea (Peres 1997, Malcolm et al. 2005), cuja produtividade pode ser 2 a 4 vezes mais alta que florestas de terra firme adjacentes (Haugaasen \& Peres 2005).

Além disso, muitas espécies de mamíferos ocorrem apenas ou preferencialmente em várzeas, como micos-de-cheiro (Saimiri spp.), uacaris (Cacajao spp.) e preguiça-de-dois-dedos (Choloepus spp.) (Ayres 1993), outras usam as várzeas em seus ciclos anuais e habitats complementares, como o peixe-boi da Amazônia, cujo ciclo de vida está diretamente associado às várzeas, onde se reproduzem e se alimentam durante os períodos de enchente e cheia, quando ocorre maior abundância de macrófitas aquáticas (da Silva et al. 2008). Indiretamente essas alterações podem afetar as duas únicas espécies de cetáceos de água doce do Novo Mundo (Inia geoffrensis e Sotalia fluviatilis) que ocorrem em maior densidade nas áreas de várzea, em função da grande diversidade e abundância de peixes quando comparadas a outras áreas da sua distribuição (Martin et al. 2004, Faustino \& da Silva 2006).

No Estado de São Paulo a extinção local do cervo-do-pantanal (Blastocerus dichotomus) está associada à destruição das várzeas pela construção de Usinas Hidrelétricas nas bacias dos Rios Paraná, Tietê, Grande e Paranapanema, bem como drenagem das várzeas para a agricultura (Piovezan et al. 2010). Em estudo realizado na área de inundação da hidrelétrica de Sérgio Motta, no Rio Paraná, Andriolo et al. (2001), Piovezan et al. (2001) e Duarte et al. (2003) estimaram uma redução populacional de $80 \%$ dois anos após o enchimento do reservatório.

\section{Impacto nas Áreas de Reserva Legal (RL)}

\section{Eliminação da exigência de RL para propriedades rurais de até quatro módulos fiscais}

As RLs têm um papel importante na paisagem para os mamíferos por dois motivos principais: 1) contribuem para manutenção da diversidade de espécies, aumentando a área de habitat disponível e criando paisagens com maior conectividade entre as populações 
remanescentes, e 2) facilitam a movimentação da fauna de maior porte funcionando como "trampolins ecológicos". Áreas de vegetação nativa protegida como RLs são fundamentais para complementar as Unidades de Conservação no seu papel de conservação da diversidade brasileira, permitindo que animais de maior porte movam-se entre grandes fragmentos de vegetação nativa através de "trampolins ecológicos" (Ribeiro et al. 2009, Cullen Jr. et al. 2003), e criando paisagens modificadas pelo homem que possam, em grande escala, diminuir o isolamento das grandes UCs.

A redução da cobertura florestal nativa tem sido particularmente impactante na Mata Atlântica onde restam menos de 16\% da cobertura florestal, sendo que somente $2 \%$ dessa área é protegida como unidades de conservação (Ribeiro et al. 2009). Muito da biodiversidade da Mata Atlântica ainda está em áreas privadas, especialmente protegidas na forma de RLs, APPs e RPPNs. A grande maioria dos fragmentos florestais existentes ( 80\%) nesse bioma é de pequena extensão ( $<50$ ha), mas dependendo da quantidade total de floresta remanescente na paisagem, muitas espécies de mamíferos podem persistir, graças a fluxos entre as populações remanescentes, facilitados pela pequena distância entre fragmentos, e pela presença de trampolins ecológicos e corredores. Nesse sentido, mesmo RLs pequenas tem um papel importante em paisagens fragmentadas, principalmente aquelas com uma baixa proporção de cobertura florestal remanescente.

Na Mata Atlântica do Planalto Paulista, um estudo recente indica que em paisagens muito desmatadas, com menos de $30 \%$ da cobertura florestal original onde, portanto, o Código Florestal atual já não é cumprido, há uma queda brusca do número de espécies de pequenos mamíferos especialistas de florestas, que é de 3 a 5 vezes menor do encontrado em paisagens com porcentuais mais altos de florestas remanescentes (Pardini et al. no prelo). Não só a maioria das espécies florestais já foram extintas, como os remanescentes destas paisagens altamente desmatadas são dominadas por espécies generalistas, como Oligoryzomys nigripes (Pardini et al. no prelo), principal reservatório do vírus responsável pela hantavirose humana na Mata Atlântica (Suzuki et al. 2004). Não por coincidência, alguns casos de hantavirose fatais em humanos têm sido registrados em áreas onde houve recente desmatamento (Silva et al. 1997). Este estudo indica que a mudança do Código Florestal e a consequente redução das RLs levarão a perdas bruscas, provavelmente não reversíveis, de biodiversidade nas paisagens modificadas pelo homem com consequências graves para serviços ecossistêmicos mediados por essa biodiversidade, como o controle do risco de doenças (Pardini et al. no prelo).

\section{Descaracterização da composição da $R L$ com a inclusão de espécies exóticas}

Plantações de espécies exóticas utilizadas como recurso madeireiro, como Eucalyptus e Pinus, geralmente abrigam uma fauna bastante depauperada. Diversos estudos têm encontrado que plantações de eucalipto abrigam um menor número de espécies de mamíferos que florestas primárias e secundárias nativas, sejam na Amazônia (Barlow et al. 2007), sejam na Mata Atlântica (Umetsu \& Pardini 2007). A descaracterização das RL acarretará em quedas populacionais e extinções locais de diversas espécies de mamíferos.

\section{Conclusão}

O não cumprimento do Código Florestal vigente certamente tem tido consequências severas não apenas para a diversidade dos mamíferos, mas também para os serviços ambientais, com repercussões na qualidade de vida humana e saúde pública. Essas consequências serão agravadas ainda mais caso as propostas legislativas de redução e descaracterização das APPs e RLs em áreas de domínio privado em todo o Brasil sejam aceitas por aprovação do Congresso Nacional. Todas as informações científicas atuais indicam a importância da manutenção das reservas legais e áreas de proteção permanente do atual Código Florestal, como medida pró-ativa para prevenir que irreversivelmente os biomas brasileiros atinjam os níveis de funcionamento e perda de diversidade biológica irreversíveis.

\section{Agradecimentos}

Nós gostaríamos de agradecer a Carlos A. Joly e Carlos A. Brocardo pelos comentários e sugestões no manuscrito. MG, RP e JMBD recebem bolsa de produtividade de pesquisa do CNPq. A FAPESP (especialmente ao programa BIOTA) pelo financiamento de parte dos estudos apresentados nesse trabalho.

\section{Referências Bibliográficas}

ABRIL, V.V., VOGLIOTTI, A., VARELA, D.M., DUARTE, J.M.B. \& CARTES, J.L. 2010. Brazilian Dwarf Brocket Deer Mazama nana (Hensel 1872). In: Neotropical cervidology: neotropical cervidology biology and medicine of Latin American deer. (J.M.B. Duarte \& S. Gonzales). Funep, Jaboticabal, IUCN, Gland, p.160-165.

ANDRIOLO, A., PIOVEZAN, U., PARANHOS DA COSTA, M.J.R., LAAKE, J. \& DUARTE, J.M.B. 2001. Estimativa aérea de abundância e distribuição do cervo do pantanal (Blastocerus dichotomus) na bacia do rio Paraná, entre as barragens de Porto Primavera e Jupiá em avaliações pré e pós enchimento da primeira cota da UHE Sérgio Motta. In: O cervo-do-pantanal de Porto Primavera: resultado de dois anos de pesquisa. (J.M.B., Duarte). Funep, 2001, 1 CD-ROM.

ARAÚJO Jr., J.P., NOGUEIRA, M.F. \& DUARTE, J.M.B. 2010. Survey for Foot-and-mouth Disease in the Endangered Marsh Deer (Blastocerus dichotomus) from Marshlands of the Paraná River Basin, Brazil. J. Wild. Dis. 46:939-943.

AYRES, J.M. 1993. As matas de várzea do Mamirauá. CNPq-Sociedade Civil Mamirauá, Brasília.

BAKER, P.J., BOITANI, L. \& HARRIS, S. 2008. Terrestrial carnivores and human food production and management. Mammal Rev. 38:123-166.

BARLOW, J., GARDNER, T.A., ARAUJO, I.S., AVILA-PIRES, T.C., BONALDO, A.B., COSTA, J.E., ESPOSITO, M.C., FERREIRA, L.V., HAWES, J., HERNANDEZ, M.M., HOOGMOED, M.S., LEITE, R.N., LO-MAN-HUNG, N.F., MALCOLM, J.R., MARTINS, M.B., MESTRE, L.A.M., MIRANDA-SANTOS, R., NUNES-GUTJAHR, A.L., OVERAL, W.L., PARRY, L., PETERS, S.L., RIBEIRO-JUNIOR, M.A., DA SILVA, M.N., MOTTA, C.D. \& PERES, C.A. 2007. Quantifying the biodiversity value of tropical primary, secondary, and plantation forests. PNAS 104:18555-18560.

CHAN, K.M.A., SHAW, M.R., CAMERON, D.R., UNDERWOOD, E.C. \& DAILY, G.C. 2006. Conservation planning for ecosystem services. Plos Biol. 4:2138-2152.

COELHO, I.P., KINDEL, A. \& COELHO, A.V.P. 2008. Roadkills of vertebrate species on two highways through the Atlantic forest Biosphere Reserve, Southern Brazil. Eur. J. Wild. Res. 54:689-699.

CULLEN Jr., L., BELTRAME, T.P., LIMA, J.F., VALLADARES-PADUA, C. \& PADUA, S.M. 2003. Trampolins ecológicos e zonas de benefício múltiplo: ferramentas agroflorestais para a conservação de paisagens rurais fragmentadas na Floresta Atlântica Brasileira. Nat. \& Conserv. 1:93-102.

DA SILVA, V.M.F., ROSAS, F.C.W. \& CATANEDE, A.M. 2008. Peixe-boi da Amazônia, Trichechus inunguis (Natterer, 1883). In: Livro vermelho da fauna brasileira ameaçada de extinção. (A.B.M. Machado, G.M. Drummond \& A.P. Paglia). Ministério do Meio Ambiente, Fundação Biodiversitas, Belo Horizonte, p.816-818.

DUARTE, J.M.B., TORRES, H.A., RAMOS H.G.C., \& LEMES, M.R.S. 2003. Efeito de uma usina hidrelétrica sobre a mortalidade de cervos-dopantanal (Blastocerus dichotomus). In Relatório Final do Projeto cervodo-pantanal de Porto Primavera. Unesp, Jaboticabal, 1 CD-ROM. 
FAUSTINO, C. \& DA SILVA, V.M.F. 2006. Seasonal use of Amazon floodplains by the tucuxi Sotalia fluviatilis (Gervais 1853), in the Central Amazon, Brazil. Latin Am. J. Aquatic Mammals, 5:95-104.

FLEURY, M. \& GALETTI, M. 2006. Forest fragment size and microhabitat effects on palm seed predation. Biol. Conserv. 131:1-13.

GALETTI, M. \& SAZIMA, I. 2006. Impact of feral dogs in an urban Atlantic forest fragment in southeastern Brazil. Nat. \& Conserv. 4:146-151.

HAUGAASEN, T. \& PERES, C.A. 2005. Mammal assemblage structure in Amazonian flooded and unflooded forests. J. Trop. Ecol. 21:133-145.

HESS, L.L., MELACK, J.M., NOVO, E.M.L.M., BARBODA, C.C.F. \& GASTIL, M. 2003. Dual-season mapping of wetland inundation and vegetation for the central Amazon basin. Rem. Sens. Environ. 87:404428.

JORDANO, P., GALETTI, M., PIZO, M.A. \& SILVA, W.R. 2006. Ligando frugivoria e dispersão de sementes à biologia da conservação. In Biologia da conservação: essências. (C.D.F. Rocha, H.D. Bergallo, M. Van Sluys \& M.A.S. Alves, ed.). Rima Editora, São Carlos, p.411-436.

LAURANCE, W.F., LAURANCE, S.G., FERREIRA, L.V., MERONA, J.M.R., GASCON, C. \& LOVEJOY, T.E. 1997. Biomass collapse in Amazonian forest fragments. Science 278:1117-1118.

LAURANCE, W.F., LOVEJOY, T.E., VASCONCELOS, H.L., BRUNA, E.M., DIDHAM, R.K., STOUFFER, P.C., GASCON, C., BIERREGAARD, R.O., LAURANCE, S.G. \& SAMPAIO, E. 2002. Ecosystem decay of Amazonian forest fragments: a 22-year investigation. Conserv. Biol. $16: 605-618$

LEES, A.C. \& PERES, C.A. 2008. Conservation value of remnant riparian forest corridors of varying quality for Amazonian birds and mammals. Conserv. Biol. 22:439-449.

LIMA, M.G. \& GASCON, C. 1999. The conservation of linear forest remnants in central Amazonia. Biol. Conserv. 91:241-247.

MACARTHUR, R.H. \& WILSON, E.O. 1967. The theory of island biogeography. Princeton University Press, New Jersey.

MACHADO, R.Z., DUARTE, J.M.B., DAGNONE, A.S. \& SZABO, M.P.J. 2006. Detection of Ehrlichia chaffeensis in Brazilian marsh deer (Blastocerus dichotomus). Vet. Parasitol. 139:262-266.

MALCOLM, J.R., PATTON, J.L. \& DA SILVA, M.N.F. 2005. Small mammal communities in upland and floodplain forests along an Amazonian white water river. Univ. Calif. Publ. Zool. 133:335-380.

MARTIN, A.R., DA SILVA, V.M.F. \& SALMON, D.L. 2004. Riverine habitat preferences of botos (Inia geoffrensis) and tucuxis (Sotalia fluviatilis) in the Central Amazon. Mar. Mammal Scien. 20:189-200.

MEESON, N., ROBERTSON, A.I. \& JANSEN, A. 2002. The effects of flooding and livestock on post-disersal seed predation in river red gum habitats. J. Appl. Ecol. 39:247-258.

METZGER, J.P. 2010. O Código Florestal tem base científica? Nat. \& Conserv. 8:1-5.

MURCIA, C. 1995. Edge effects in fragmented forests: implications for conservation. TREE 10:58-62.

NAXARA, L. 2008. Importância dos corredores ripários para a fauna pequenos mamíferos em manchas de floresta, matriz do entorno e elementos lineares em uma paisagem fragmentada de Mata Atlântica. Dissertation, Universidade de São Paulo, São Paulo.

NICHOLS, E., GARDNER, T.A., PERES, C.A. \& SPECTOR, S. 2009. Codeclining mammals and dung beetles: an impending ecological cascade. Oikos 118:481-487.

OWEN, R.D., GOODIN, D.G., KOCH, D.E., CHU, Y.-K. \& JONSSON, C.B. 2010. Spatiotemporal variation in Akodon montensis (Cricetidae: Sigmodontinae) and hantaviral seroprevalence in a subtropical forest ecosystem. J. Mammal. 91:467-481.

PARDINI, R., DE SOUZA, S.M., BRAGA-NETO, R. \& METZGER, J.P. 2005. The role of forest structure, fragment size and corridors in maintaining small mammal abundance and diversity in an Atlantic forest landscape. Biol. Conserv. 124:253-266.

PARDINI R, BUENO AA, GARDNER TA, PRADO PI, METZGER JP, 2010 Beyond the Fragmentation Threshold Hypothesis: Regime Shifts in Biodiversity Across Fragmented Landscapes. PLoS ONE 5(10): e13666. doi:10.1371/journal.pone.0013666
PEREIRA, M.C., SZABÓ, M.P.J., BECHARA, G.H., MATUSHIMA, E.R., DUARTE, J.M.B., RECHAV, Y., FIELDEN, L. \& KEIRANS, J. 2000 Ticks (Acari; Ixodidae) associated with wild animals in the Pantanal region of Brazil. J. Med. Entomol., 37:979-983.

PERES, C.A. 1997. Primate community structure at twenty western Amazonian flooded and unflooded forests. J. Trop. Ecol. 13:381-405.

PIOVEZAN, U., ANDRIOLO, A., JACOB, A.A., RAMOS, H.G.C., PARANHOS DA COSTA, M.J.R. \& DUARTE, J.M.B. 2001. Caracterização das reações do cervo-do-pantanal (Blastocerus dichotomus) ao impacto causado pelo enchimento parcial do reservatório da usina hidrelétrica Sérgio Motta (porto primavera). In O cervo-dopantanal de Porto Primavera: resultado de dois anos de pesquisa (J.M.B Duarte). FUNEP, 2001. 1 CD-ROM.

PIOVEZAN, U., TIEPOLO, L.M., TOMAS, W.M., DUARTE, J.M.B., VARELA, D., \& MARINHO FILHO, J.S. 2010. Marsh Deer Blastocerus dichotomus (Illiger 1815). In Neotropical cervidology: biology and medicine of latin american deer (J.M.B. Duarte \& S. Gonzáles). Funep, Jaboticabal, IUCN, Gland, p.66-76.

RAMIREZ-MARCIAL, N., GONZALES-ESPINSA, M. \& WILLIANSLINERA, G. 2001. Antropogenic disturbance and tree diversity in montane rain forest in Chiapas, Mexico. For. Ecol. Manag. 154:311-326.

REIS, N.R., PERACHI, A.L., PEDRO, W.A. \& LIMA, I.P. 2006. Mamíferos do Brasil. Editora da Universidade de Londrina, Londrina.

REYS, P., SABINO, J. \& GALETTI, M. 2009. Frugivory by the fish Brycon hilarii (Characidae) in western Brazil. Acta Oecol. 35:136-141.

RIBEIRO, M.C., METZGER, J.P., MARTENSEN, A.C., PONZONI, F.J. \& HIROTA, M.M. 2009. The Brazilian Atlantic Forest: how much is left, and how is the remaining forest distributed? Implications for conservation. Biol. Conserv. 142:1141-1153.

SAZIMA, I., FISCHER, W.A., SAZIMA, M. \& FISCHER, E.A. 1994. The fruit bat Artibeus lituratus as a forest and city dweller. Ciênc. e Cult. 46:164-168.

SAZIMA, M., FABIÁN, M.E. \& SAZIMA, I. 1982. Polinização de Luehea speciosa (Tiliaceae) por Glossophaga soricina (Chiroptera, Phyllostomidae). Rev. Brasil. Biol. 42:505-513.

SCHIPPER, J., CHANSON, J.S., CHIOZZA, F., COX, N.A., HOFFMANN, M., KATARIYA, V., LAMOREUX, J., RODRIGUES, A.S.L., STUART, S.N., TEMPLE, H.J., BAILLIE, J., BOITANI, L., LACHER, T.E., MITTERMEIER, R.A., SMITH, A.T., ABSOLON, D., AGUIAR, J.M., AMORI, G., BAKKOUR, N., BALDI, R., BERRIDGE, R.J., BIELBY, J., BLACK, P.A., BLANC, J.J., BROOKS, T.M., BURTON, J.A., BUTYNSKI, T.M., CATULLO, G., CHAPMAN, R., COKELISS, Z., COLLEN, B., CONROY, J., COOKE, J. G., DA FONSECA, G.A.B., DEROCHER, A.E., DUBLIN, H.T., DUCKWORTH, J.W., EMMONS, L., EMSLIE, R.H., FESTA-BIANCHET, M., FOSTER, M., FOSTER, S., GARSHELIS, D.L., GATES, C., GIMENEZ-DIXON, M., GONZALEZ, S., GONZALEZ-MAYA, J.F., GOOD, T.C., HAMMERSON, G., HAMMOND, P.S., HAPPOLD, D., HAPPOLD, M., HARE, J., HARRIS R.B., HAWKINS, C.E., HAYWOOD, M., HEANEY, L.R., HEDGES, S., HELGEN, K.M., HILTON-TAYLOR, C., HUSSAIN, S.A., ISHII, N., JEFFERSON, T.A., JENKINS, R.K.B., JOHNSTON, C.H., KEITH, M., KINGDON, J., KNOX, D. H., KOVACS, K.M., LANGHAMMER, P., LEUS, K., LEWISON, R., LICHTENSTEIN, G., LOWRY, L.F., MACAVOY,Z., MACE, G.M., MALLON, D.P., MASI, M., MCKNIGHT, M.W., MEDELLIN, R.A., MEDICI, P., MILLS, G., MOEHLMAN, P.D., MOLUR, S., MORA, A., NOWELL AND, K., OATES, J.F., OLECH, W., OLIVER, W.R.L., OPREA, M., PATTERSON, B.D., PERRIN, W.F., POLIDORO, B.A., POLLOCK, C., POWEL, A., PROTAS, Y., RACEY, P., RAGLE, J., RAMANI, P., RATHBUN, G., REEVES, R.R., REILLY, S.B., REYNOLDS, J.E., RONDININI, C., ROSELL-AMBAL, R.G., RULLI, M., RYLANDS, A.B., SAVINI, S., SCHANK, C.J., SECHREST, W., SELF-SULLIVAN, C., SHOEMAKER, A., SILLERO-ZUBIRI, C., DE SILVA, N., SMITH, D.E., SRINIVASULU, C., STEPHENSON, P.J., VAN STRIEN, N., TALUKDAR, B.K., TAYLOR, B.L., TIMMINS, R., TIRIRA, D.G., TOGNELLI, M.F., TSYTSULINA, K., VEIGA, L.M., VIE, J.C., WILLIAMSON, E.A., WYATT, S.A., XIE, Y. \& YOUNG, B.E. 2008. The status of the world's land and marine mammals: diversity, threat, and knowledge. Science 322:225-230. 
SILVA, A., VASCONCELOS, M.J., HIDALGO, N.T.R., VEIGA, A.P.R., CANZIAN, M., MAROTTO, P.C.F. \& LIMA, V.C.P. 1997. Hantavirus pulmonary syndrome: report of the first three cases in São Paulo, Brazil. Rev. Instit. Med. Trop. São Paulo 39:231-234.

SPAROVEK, G., RANIERI, S.B.L., GASSNER, A., DE MARIA, I.C., SCHNUG, E., DOS SANTOS, R.F. \& JOUBERT, A. 2002. A conceptual framework for the definition of the optimal width of riparian forests. Agr. Ecosyst. \& Environ. 90:169-175.

STENSETH, N.C., LEIRS, H., SKONHOFT, A., DAVIS, S.A., PECH, R.P., ANDREASSEN, H.P., SINGLETON, G.R., LIMA, M., MACHANG'U, R.S., MAKUNDI, R.H., ZHANG, Z., BROWN, P.R., SHI, D. \& WAN, X. 2003. Mice, rats, and people: the bio-economics of agricultural rodent pests. Front. Ecol. Environ. 1: 367-375.

SUZUKI, A., BISORDI, I., LEVIS, S., GARCIA, J., PEREIRA, L.E., SOUZA, R.P., SUGAHARA, T.K.N., PINI, N., ENRIA, D. \& SOUZA, L.T.M. 2004. Identifying rodent hantavirus reservoirs, Brazil. Emer. Infect. Dis. 10:2127-2134.

SZABÓ, M.P.J., LABRUNA, M.B., PEREIRA, M.C. \& DUARTE, J.M.B. 2003. Ticks (Acari: Ixodidae) on Wild Marsh deer (Blastocerus dichotomus) from Southeast Brazil: infestations before and after habitat loss. J. Med. Ent. 40:268-274.

SZABÓ, M.P.J., BOTELHO, M.C., RAMOS, H.G.C., GARCIA, M., CASTAGNOLLI, K., PINTER, A. VERONEZ, V., MAGALHAES, G., DUARTE, J.M.B. \& LABRUNA, M.B. 2007. Species diversity and seasonality of free-living ticks (Acari: Ixodidae) in the natural habitat of wild Marsh deer (Blastocerus dichotomus) in Southeastern Brazil. Vet. Parasitol., 143:147-154.

SZABÓ, M.P.J., LABRUNA, M.B., GARCIA, M., PINTER, A., CASTAGNOLLI, K., CASTRO, M.B., VERONEZ, V., MAGALHAES, G., VOGLIOTTI, A., \& DUARTE, J.M.B. 2009. Ecological aspects of the free-living ticks (Acari: Ixodidae) on animal trails within Atlantic rainforest in south-eastern Brazil. Ann. Trop. Med. Parasitol. 103:57-72
TABARELLI, M. \& PERES, C.A. 2002. Abiotic and vertebrate seed dispersal in the Brazilian Atlantic forest: implications for forest regeneration. Biol. Conserv. 106:165-176.

TERBORGH, J., LOPEZ, L., NUNEZ, P., RAO, M., SHAHABUDDIN, G., ORIHUELA, G., RIVEROS, M., ASCANIO, R., ADLER, G.H., LAMBERT, T.D. \& BALBAS, L. 2001. Ecological meltdown in predatorfree forest fragments. Science 294:1923-1926.

TIEMANN, J.C.H., SOUZA, S.L.P., RODRIGUES, A.A.R., DUARTE, J.M.B. \& GENNARI, S.M. 2005. Environmental effect on the occurrence of antiNeospora caninum antibodies in pampas deer (Ozotoceros bezoarticus). Vet. Parasitol. 134:73-76.

UMETSU, F. \& PARDINI, R. 2007. Small mammals in a mosaic of forest remnants and anthropogenic habitats-evaluating matrix quality in an Atlantic forest landscape. Lands. Ecol. 22:517-530.

VIÉ, J.C., HILTON-TAYLOR, C. \& STUART, S.N. 2009. Wildlife in a changing world - an analysis of the 2008 IUCN Red List of threatened species. IUCN, Gland, Switzerland.

VIEIRA, E.M. \& MARINHO-FILHO, J. 1998. Pre- and post-fire habitat utilization by rodents of Cerrado from Central Brazil. Biotropica 30:491-496.

VIEIRA, E.M. \& MONTEIRO-FILHO, E.L.A. 2003. Vertical stratification of small mammals in the Atlantic rain forest of South-eastern Brazil. J. Trop. Ecol. 19:501-507.

VOGLIOTTI, A. \& DUARTE, J.M.B. 2009. Discovery of the first wild population of the small red brocket deer Mazama bororo (ArtiodactylaCervidae). Mastozool. Neotrop. 16:499-503.

VOGLIOTTI, A. \& DUARTE, J.M.B. 2010. Small Red Brocket Deer Mazama bororo (Duarte 1996) In Neotropical cervidology, biology and medicine of latin american deer. Funep, Jaboticabal, IUCN, Gland, p.172-176. 